\title{
The Ground State Rotational Constants of $\mathrm{H}_{3} \mathrm{SiD}$
}

\author{
H. Bürger and A. Rahner \\ FB 9 - Anorganische Chemie, Universität-Gesamthochschule, Wuppertal, West Germany \\ J. Kauppinen \\ Physics Department, University of Oulu, SF-90570 Oulu, Finland
}

Z. Naturforsch. 40 a, 386-388 (1985); received December 24, 1984

The $J$-dependent rotational constants up to sextic coefficients of $\mathrm{H}_{3}{ }^{28} \mathrm{SiD}$ were determined by a fit $\left(\sigma=3.5 \times 10^{-4} \mathrm{~cm}^{-1}\right)$ of 1505 ground state combination differences formed from transitions of the $v_{3}, v_{5}$, and $v_{6}$ vibrational fundamentals which were measured with a resolution of $0.005 \mathrm{~cm}^{-1}$. The $K$-dependent constants $A_{0}$ and $D_{K}^{0}$ were obtained from perturbation-allowed transitions observed within $v_{6}$.

The ground state constants of the prolate symmetric top $\mathrm{H}_{3}{ }^{28} \mathrm{SiD}$ have been determined from rovibrational transitions in the $v_{1} / v_{4}$ region near $2200 \mathrm{~cm}^{-1}$, measured with a grating instrument with a resolution of 0.030 and an accuracy of ca. $0.004 \mathrm{~cm}^{-1}[1] . B_{0}, D_{J}^{0}$ and $D_{J K}^{0}$ were determined by means of 417 ground state combination differences which were fitted with a standard deviation of $3.9 \times 10^{-3} \mathrm{~cm}^{-1}$, while the quantity $\left(A_{0}-B_{0}-5 D_{K}^{0}\right)$ was deduced from perturbation-allowed transitions [2]. Combination of these and allowed transitions provided combination differences between the $K=1$ and $K=2$ ground state levels. Since $A_{0}$ and $D_{K}^{0}$ could not be separated from these differences, $D_{K}^{0}$ was constrained to the value listed in Table 1.

In context with the rovibrational analysis of the $v_{3} / v_{5} / v_{6}$ bands spanning the 660 to $1070 \mathrm{~cm}^{-1}$ region, we have recorded high resolution spectra with the FT spectrometer at the University of Oulu. The resolution was $0.0045 \mathrm{~cm}^{-1}$, which combines with the Doppler width $\left(2 \times 10^{-3} \mathrm{~cm}^{-1}\right.$ at $\left.1000 \mathrm{~cm}^{-1}\right)$ and the pressure broadening at $2.7 \mathrm{mbar}$ to yield an experimental fwmh of $\sim 0.005 \mathrm{~cm}^{-1}$. Calibration was with $\mathrm{CO}_{2}$ lines [3]; wavenumber accuracy $<1 \times 10^{-4} \mathrm{~cm}^{-1}$. About 3000 transitions belonging to $v_{3}\left(912.9 \mathrm{~cm}^{-1}\right), v_{5}\left(951.4 \mathrm{~cm}^{-1}\right)$ and $v_{6}\left(784.3 \mathrm{~cm}^{-1}\right)$ were assigned. A total of 1505 ground state combination differences were formed from pairs of normally allowed, unit-weighted lines with $\Delta J=+1$ and $0,+1$ and -1 and 0 and -1 respectively, all reaching the same upper state. Lines which were

Reprint requests to Prof. Dr. H. Bürger, Universität-Gesamthochschule, Gaußstraße 20, Postfach 100127 , D-5600 Wuppertal 1. either too broad or too strong and obviously composites of several components were not used in the refinement. Of these pairs, 292, 527 and 686 belonged to $v_{3}, v_{5}$ and $v_{6}$ respectively with $J_{\max }$ and $K_{\max }=21$ and 16 respectively. The combination differences were fitted to yield the coefficients in the $J$-dependent terms of the rotational term value expression (1):

$$
\begin{aligned}
E_{0}(J, K)= & \left(A_{0}-B_{0}\right) K^{2}+B_{0} J(J+1) \\
& -D_{J}^{0} J^{2}(J+1)^{2}-D_{J K}^{0} J(J+1) K^{2} \\
& -D_{K}^{0} K^{4}+H_{J}^{0} J^{3}(J+1)^{3} \\
& +H_{J K}^{0} J^{2}(J+1)^{2} K^{2} \\
& +H_{K J}^{0} J(J+1) K^{4}+H_{K}^{0} K^{6} .
\end{aligned}
$$

The results are listed in Table 1. It is obvious that the $J$-dependent sextic coefficients are required to

Table 1. Ground state constants of $\mathrm{H}_{3}{ }^{28} \mathrm{SiD}\left(\mathrm{cm}^{-1}\right)$.

\begin{tabular}{llll}
\hline & Ref. [1, 2] & \multicolumn{2}{c}{ This study } \\
\hline$A_{0}$ & 2.863 34(45) & \multicolumn{2}{c}{$2.863424(40)$} \\
$D_{K}^{0} \times 10^{5}-3.85^{\text {a }}$ & \multicolumn{2}{c}{$-3.82(29)$} \\
$B_{0}$ & $2.099477(11)$ & $2.0994837(19)$ & $2.0995687(11)$ \\
$D_{J}^{0} \times 10^{5}$ & $1.5644(20)$ & $1.5645(3)$ & $1.5917(4)$ \\
$D_{J K}^{0} \times 10^{5}$ & $5.237(9)$ & $5.2462(15)$ & $5.3795(19)$ \\
$H_{J}^{0} \times 10^{10}-$ & - & $2.10(5)$ \\
$H_{J K}^{0} \times 10^{9}$ & - & - & $2.89(3)$ \\
$H_{K J}^{0} \times 10^{9}$ & - & - & $-1.45(7)$ \\
No. of dif- & & & 1505 \\
ferences & 417 & 1505 & 0.35 \\
$\sigma \times 10^{3}$ & 3.9 & 1.08 &
\end{tabular}

a $D_{K}^{e}$ from harmonic force field.

0340-4811/85/0400-0386\$01.30/0. - Please order a reprint rather than making your own copy. 


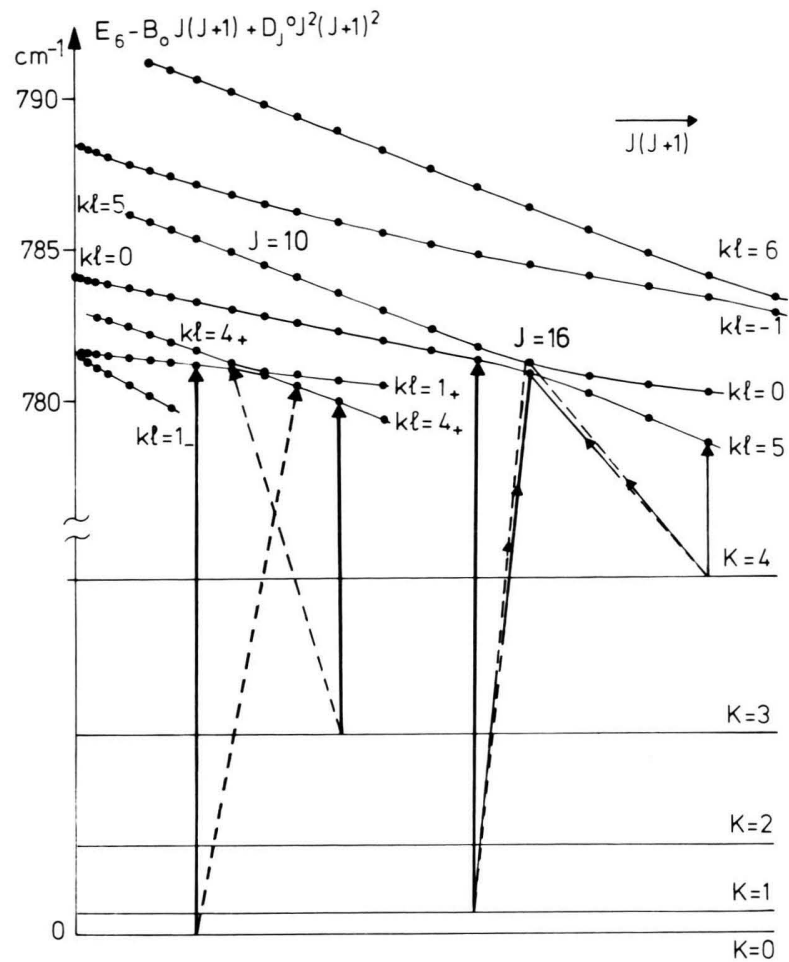

Fig. 1. Reduced upper state and schematic ground state energies of $\mathrm{H}_{3}{ }^{28} \mathrm{SiD}$. Normally allowed transitions are marked by full lines, perturbation-allowed transitions (only schematically) by broken lines. Note that for the transitions reaching the $J^{\prime}=16$ doublet normally and perturbation-allowed transitions cannot be distinguished.

reproduce the experimental data to an accuracy corresponding to that of the measurement.

After the $J$-dependent coefficients had been calculated, the $K$-dependent constants $A_{0}$ and $D_{K}^{0}$ were determined in an independent second step. The $k l>0$ and $k l<0$ subsystems of $v_{6}$ undergo $\Delta l= \pm 2$, $\Delta k= \pm 2$ and $\Delta l= \pm 2, \Delta k=\mp 1$ interactions. These provoke level crossings of $(k l), k l<0$ and $(k l)+5, k l>0$ levels at $J=10,16$ and 20 for $|k-l|=0,1$ and 2 , respectively. Close to these crossings, $\Delta l= \pm 2, \Delta k= \pm 5$ interactions which are allowed by the Amat rule lead to perturbationallowed transitions with $\Delta k=-2$ and $\Delta k= \pm 4$. These were observed near to the level crossings at $J^{\prime}=10$ and 16 respectively while the respective transitions near to the crossing at $J^{\prime}=20$ were too weak to be measured.

Of the (split) $k l=4\left(\mathrm{~A}_{1} \mathrm{~A}_{2}\right)$ level, only the $A_{+}$ component interacts with the attached $A_{+}$component of the $k l=1$ sublevel. The $J=16,(k=0$, $l=-1)$ and $(k=5, l=+1)$ states are so close that the normally allowed $\Delta k= \pm 1$ and perturbationallowed $\Delta k=-2$ and $\Delta k=+4$ transitions occur with apparently equal intensity as a doublet with a splitting of $0.2484(7) \mathrm{cm}^{-1}$.

Reduced upper state energies of relevant sublevels and transitions are illustrated in Figure 1. The observed perturbation-allowed transitions and representative, normally-allowed transitions which were employed to form $\Delta K^{\prime \prime}=3$ ground state combination differences are listed in Table 2. From these differences, the relations $9\left(A_{0}-9 D_{K}^{0}\right)$ and $15\left(A_{0}-17 D_{K}^{0}\right)$ were obtained by means of the coefficients of the $J$-dependent terms, and these allowed $A_{0}$ and $D_{K}^{0}$ to be determined. The present ground state constants, if calculated to the same order, agree with the previously reported data $[1,2]$ to

Table 2. Perturbation-allowed and attached normally allowed transitions of $v_{6}$ of $\mathrm{H}_{3}{ }^{28} \mathrm{SiD}\left[\mathrm{cm}^{-1}\right]$.

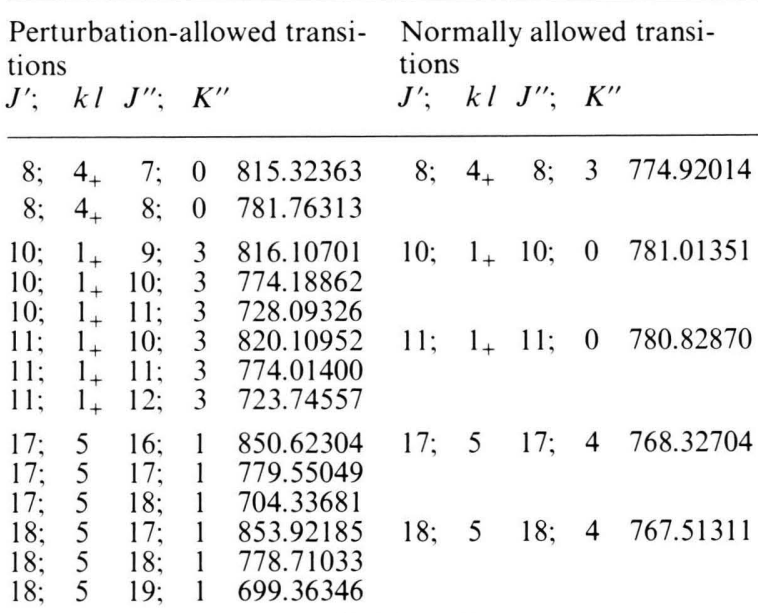

\begin{tabular}{ccccll}
\hline \multicolumn{2}{c}{ Transitions } \\
$J^{\prime} ;$ & $k l$ & $J^{\prime \prime} ;$ & $K^{\prime \prime}$ & & $\begin{array}{l}\text { Upper state } \\
\text { energies }\end{array}$ \\
\hline $16 ;$ & $5 / 0$ & $15 ;$ & 4 & $836.16447 /$ & $1351.21211 /$ \\
& & & & 835.91607 & 1350.96371 \\
$16 ;$ & $5 / 0$ & $16 ;$ & 4 & $769.26436 /$ & $1351.21193 /$ \\
& & & & 769.01607 & 1350.96364 \\
$16 ;$ & $5 / 0$ & $17 ;$ & 4 & $698.21837 /$ & $1351.21189 /$ \\
& & & & 697.97044 & 1350.96396 \\
$16 ;$ & $5 / 0$ & $15 ;$ & 1 & $847.44121 /$ & $1351.21208 /$ \\
& & & & 847.19347 & 1350.96426 \\
$16 ;$ & $5 / 0$ & $16 ;$ & 1 & $780.51419^{\text {a }} /$ & $1351.21011 /$ \\
& & & & 780.26855 & 1350.96439 \\
$16 ;$ & $5 / 0$ & $17 ;$ & 1 & $709.44508^{\text {a/ }}$ & $1351.21355 /$ \\
& & & & 709.19562 & 1350.96401
\end{tabular}

\footnotetext{
a Weakly blended.
} 
within three of their standard deviations. Due to the correlation with the $H$ 's, $B_{0}$ and the $D$ 's change when allowance is made in the fit for sextic coefficients.

In conclusion, due to the accuracy of the measurements, which has been improved by at least an order of magnitude, and the larger number of combination differences determined, the precision of the ground state constants has been enhanced in the present study by one order of magnitude. The sextic

[1] W. B. Olson and R. W. Lovejoy, J. Mol. Spectrosc. 66, 314 (1977).

[2] R. W. Lovejoy and W. B. Olson, J. Chem. Phys. 57, 2224 (1972). constants and $D_{K}^{0}$ have been determined experimentally for the first time. Finally, no indication for ground state perturbations was found.

Acknowledgements

Dr. G. Graner is thanked for valuable discussions. Support by the Deutsche Forschungsgemeinschaft through the SFB42 is gratefully acknowledged.

[3] J. Kauppinen, K. Jolma, and V. M. Horneman, Appl. Optics 21, 3332 (1982). 